\title{
Comunicação/Communication
}

\section{Série de casos agudos de doença de Chagas atendidos num serviço terciário de Manaus, Estado do Amazonas, de 1980 a 2006}

\author{
Series of acute Chagas' disease cases attended at a tertiary-level clinic in Manaus, State of \\ Amazonas, from 1980 to 2006
}

\author{
Wuelton Marcelo Monteiro ${ }^{1,2}$, Maria das Graças Vale Barbosa ${ }^{1,3,4}$, Max Jean de Ornelas Toledo ${ }^{5}$, Flávio Augusto Fé ${ }^{4}$ \\ e Nelson Ferreira Fé ${ }^{4}$
}

\begin{abstract}
RESUMO
Introdução: A doença de Chagas é um problema emergente e negligenciado na Região Amazônica. Métodos: Descreve-se uma série de casos agudos autóctones de doença de Chagas atendidos na Fundação de Medicina Tropical do Amazonas, Manaus, de 1980 a 2006. Resultados: Registraram-se 29 casos, sendo 19 do sexo masculino e 10 casos do sexo feminino. Quinze eram casos isolados e 14 provenientes de surtos. Os sinais/sintomas mais freqüentes foram febre, fadiga, cefaléia, mialgia, calafrios, palidez, dispnéia e edema de face e de membros inferiores. Não foi registrado nenhum óbito. Conclusões: A doença incidiu com frequência em jovens. Os métodos parasitológicos mostraram elevada sensibilidade.
\end{abstract}

Palavras-chaves: Doença de Chagas. Casos agudos. Estado do Amazonas.

\begin{abstract}
Introduction: Chagas disease is an emerging and neglected problem in the Brazilian Amazon region. Methods: This study describes a series of acute autochthonous cases of Chagas disease that were attended at the Tropical Medicine Foundation of Amazonas, Manaus, between 1980 and 2006 Results: Twenty-nine cases were recorded: 19 (65.5\%) were male and $10(34.5 \%)$ cases were female. Fifteen $(51.7 \%)$ were isolated cases and 14 (48.3\%) were from outbreaks. The commonest signs and symptoms were fever, fatigue, headache, myalgia, chills, pallor, dyspnea and edema of the face and lower limbs. No deaths were recorded. Conclusions: The disease occurred frequently among young people. The parasitological methods showed high sensitivity.
\end{abstract}

Key-words: Chagas disease. Acute cases. State of Amazonas.

1. Programa de Pós-Graduação em Medicina Tropical, Universidade do Estado do Amazonas, Fundação de Medicina Tropical do Amazonas, Manaus, AM. 2. Departamento de Saúde Coletiva, Universidade Federal do Amazonas, Manaus, AM. 3. Centro Universitário Nilton Lins, Manaus, AM. 4. Gerência de Entomologia e Leishmaniose, Fundação de Medicina Tropical do Amazonas, Manaus, AM 5. Departamento de Ciências Básicas da Saúde, Universidade Estadual de Maringá, Maringá, PR.

Endereço para correspondência: Prof. Wuelton Marcelo Monteiro. Programa de PósGraduação em Medicina Tropical da Universidade do Estado do Amazonas, Manaus, AM. Av. Pedro Teixeira 25, Dom Pedro, 69040-000 Manaus, AM.

Fax: 5592 3656-8269

e-mail: wueltonmm@ibest.com.br

Recebido para publicação em 15/11/2009

Aceito em 23/02/2010
Por muito tempo considerada hipoendêmica na Região Amazônica, a doença de Chagas recentemente vem sendo reconhecida como um problema emergente e negligenciado nesta região, com centenas de casos descritos nas últimas décadas, a maioria deles no Estado do Pará, em microepidemias ou em casos isolados ${ }^{1}$. Informações do Ministério da Saúde ${ }^{2}$ indicam que de 2005 a maio 2009 foram notificados 455 casos de doença de Chagas aguda no Brasil, dos quais 389 (85,5\%) ocorreram na Região Norte do país. Neste período, os estados brasileiros com maior número de casos registrados foram o Pará, com 310 (68,1\%) casos e o Amazonas, $\operatorname{com} 29(6,4 \%)^{2}$.

O Inquérito Sorológico $\mathrm{Nacional}^{3}$ estimou uma prevalência de 1,9\% para o Estado do Amazonas, o que proporcionava um número esperado de 8.195 casos positivos para uma população de 435.379 habitantes à época. Neste estado, foram encontrados dois casos de miocardiopatia dilatada grave com sorologia positiva para infecção chagásica, os quais evoluíram para o óbito ${ }^{4}$ e três casos de insuficiência cardíaca, com características clínicas, eletrocardiográficas e ecocardiográficas sugestivas de cardiopatia chagásica crônica, cuja sorologia foi positiva para Trypanosoma $\mathrm{cruzi}^{5}$. Recentemente, um inquérito realizado na área rural de Manaus detectou uma taxa de 1,3\% de indivíduos com sorologia positiva para a infecção pelo T. cruzi $i^{6}$. Porém, não existem informações sobre a casuística da doença aguda nos serviços de saúde do Estado do Amazonas, o que motivou a realização deste estudo, que descreve os aspectos clínico-epidemiológicos dos casos agudos autóctones de doença de Chagas atendidos na Fundação de Medicina Tropical do Amazonas, de 1980 a 2006.

A Fundação de Medicina Tropical do Amazonas (FMT-AM) é um serviço terciário especializado em doenças infecciosas e parasitárias, localizado em Manaus, de referência nesta área para o Estado do Amazonas, Brasil. Oferece atendimento a pacientes referenciados por diversas unidades públicas e privados de Manaus e do interior do estado. Este trabalho, retrospectivo do tipo série de casos, foi conduzido por meio da análise dos prontuários e de livros de registro de exames dos pacientes com doença de Chagas aguda, atendidos entre 1980 e 2006 nessa instituição. Foram considerados casos agudos aqueles que apresentaram exames parasitológicos diretos (a fresco, gota espessa ou método de Strout) e/ou $\operatorname{IgM}$ antiT. cruzi positivos no teste de hemaglutinação, ELISA e/ou imunofluorescência (títulos $\geq 40$ foram considerados positivos). 
O instrumento de coleta dos dados incluiu a busca por informações demográficas (idade, sexo, município de procedência, zona de moradia - rural ou urbana -, tipo de moradia e ocupação principal), clínico-laboratoriais (sinais e sintomas, resultados de exames laboratoriais) e provável via de transmissão. Para o tratamento, os pacientes receberam benzonidazol, em esquema variável de 30 a 60 dias, em doses de 4 a $10 \mathrm{mg} / \mathrm{kg} / \mathrm{dia}$. Infelizmente, não haviam registros de informações sobre o seguimento pósterapêutico dos pacientes, já que estes são na maioria do interior do estado e retornaram ao município de residência após instituição do tratamento.

\section{Ética}

O estudo foi aprovado pelo Comitê de Ética em Pesquisa da Fundação de Medicina Tropical do Amazonas.

Foram registrados 29 casos agudos de doença de Chagas na FMT-AM no período do estudo. Destes, 19 (65,5\%) eram do sexo masculino, com média etária de 17,9 anos, variando de 2 a 29 anos. Registraram-se 10 (34,5\%) casos do sexo feminino, com média etária de 27,3 anos, variando de 4 a 68 anos. Do total de casos registrados, $15(51,7 \%)$ eram casos isolados e $14(48,3 \%)$ apareceram na forma de surtos, sendo três casos do município de São Paulo de Olivença em 1985, nove de Tefé em 2004 e dois de Iranduba em 1992 (Tabela 1). Os municípios com registro de casos foram Tefé (11), São Paulo de Olivença (4), Manaus (4), Iranduba (3), Anamã (1), Barcelos (1), Carauari (1), Coari (1), Manacapuru (1), Maraã (1) e Tabatinga (1) (Figura 1).

Foram observados $18(62,1 \%)$ casos com moradia na zona rural e $11(37,9 \%)$ na zona urbana. As ocupações informadas pelos casos foram as de agricultor ( 7 casos), estudante (7), extrativista (7), do lar (3), bancário (1) e militar (1). Os tipos de moradia predominantes foram as casas de madeira com telha (8 casos), moradias precárias de taipa (7), madeira com cobertura de palha (6), madeira com cobertura de zinco (4) e alvenaria (3).

Os pacientes adultos reconheceram exemplares de barbeiros, entre eles Rhodnius brethesi em Barcelos, Rhodnius pictipes em São Paulo de Olivença, Carauari e pelo paciente de Manaus que se
TABELA 1 - Município de origem, sexo, idade e exames que confirmaram a fase aguda da doença de Chagas em pacientes atendidos na Fundação de Medicina Tropical do Amazonas, de 1980 a 2006.

\begin{tabular}{|c|c|c|c|c|c|}
\hline Caso & Ano & $\begin{array}{l}\text { Município } \\
\text { de origem }\end{array}$ & Sexo & $\begin{array}{l}\text { Idade } \\
\text { (anos) }\end{array}$ & $\begin{array}{c}\text { Exames confirmatórios } \\
\text { da fase aguda }\end{array}$ \\
\hline 1 & 1980 & São Paulo de Olivença & $\mathrm{F}$ & 4 & GE, EF, TS \\
\hline 2 & 1981 & Manaus & M & 26 & GE,EF \\
\hline 3 & 1983 & Barcelos & M & 24 & GE \\
\hline 4 & 1985 & São Paulo de Olivença & M & 10 & GE, EF, MS \\
\hline 5 & 1985 & São Paulo de Olivença & M & 12 & GE, EF, MS \\
\hline 6 & 1985 & São Paulo de Olivença & M & 12 & GE \\
\hline 7 & 1990 & Tefé & $\mathrm{F}$ & 6 & GE,EF \\
\hline 8 & 1990 & Tefé & M & 10 & GE,EF \\
\hline 9 & 1991 & Iranduba & M & 10 & GE, EF, MS, TS \\
\hline 10 & 1992 & Iranduba & M & 2 & GE, EF, MS, TS \\
\hline 11 & 1992 & Iranduba & M & 4 & GE, EF, MS, TS \\
\hline 12 & 1993 & Carauari & $\mathrm{F}$ & 12 & GE, EF, MS, TS \\
\hline 13 & 1994 & Manaus & M & 25 & GE, EF, MS, TS \\
\hline 14 & 1999 & Tabatinga & M & 29 & GE, EF, MS, TS \\
\hline 15 & 2003 & Coari & M & 4 & GE, EF, MS, TS \\
\hline 16 & 2004 & Manaus & M & 27 & GE, EF, MS, TS \\
\hline 17 & 2005 & Tefé & $\mathrm{F}$ & 16 & GE, EF, MS, TS \\
\hline 18 & 2005 & Tefé & $\mathrm{F}$ & 22 & GE, EF, MS \\
\hline 19 & 2005 & Tefé & $\mathrm{F}$ & 25 & GE \\
\hline 20 & 2005 & Tefé & $\mathrm{F}$ & 64 & GE, EF, MS \\
\hline 21 & 2005 & Tefé & $\mathrm{F}$ & 68 & GE, EF, MS \\
\hline 22 & 2005 & Tefé & M & 14 & GE, EF, MS \\
\hline 23 & 2005 & Tefé & M & 24 & GE, EF, MS, TS \\
\hline 24 & 2005 & Tefé & M & 27 & GE, EF, MS, TS \\
\hline 25 & 2005 & Tefé & M & 29 & GE, EF, MS, TS \\
\hline 26 & 2005 & Manacapuru & M & 22 & GE, EF, MS, TS \\
\hline 27 & 2006 & Anamã & $\mathrm{F}$ & 42 & GE \\
\hline 28 & 2006 & Manaus & M & 29 & GE \\
\hline 29 & 2006 & Maraã & $\mathrm{F}$ & 14 & GE, TS \\
\hline
\end{tabular}

F: feminino, M: masculino, GE: gota espessa, EF: exame a fresco, MS: método de Strout, TS: testes sorológicos IgM antiT. cruzi.

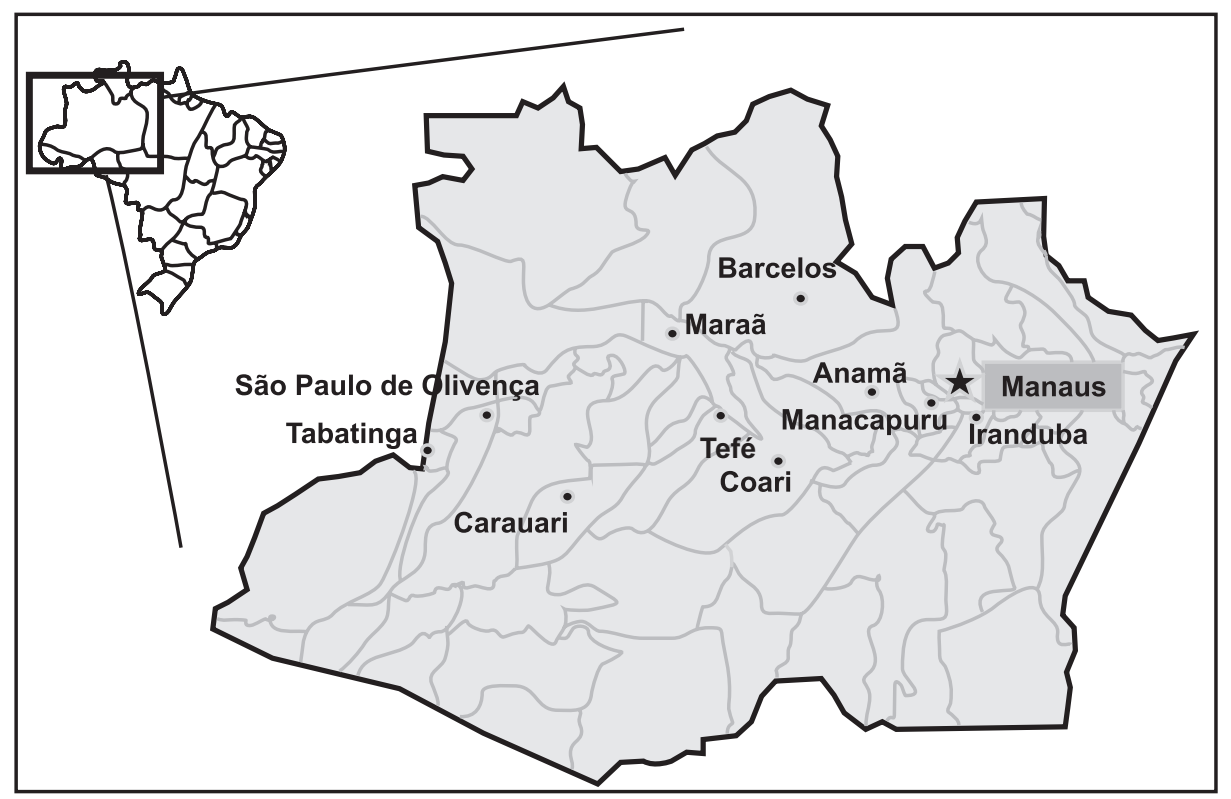

FIGURA 1 - Municípios do Estado do Amazonas com registro de casos agudos de doença de Chagas atendidos na Fundação de Medicina Tropical do Amazonas, de 1980 a 2006. 
infectou na região do Purus, e Rhodnius robustus em Manaus e São Paulo de Olivença.

Os percentuais de positividade, conforme o método empregado para o diagnóstico etiológico, foram de $100 \%$ (29/29), 82,1\% (23/28), $79,2 \%$ (19/24), $75 \%$ (15/20), 100\% (24/24) e $100 \%$ $(20 / 20)$, respectivamente para os exames de gota espessa, exame direto a fresco, método de Strout, IgM antiT. cruzi, xenodiagnóstico e hemocultura. Foram demonstrados anticorpos IgG antiT. cruzi, em $80 \%$ dos casos submetidos aos testes sorológicos. Todos os pacientes tiveram seu diagnóstico confirmado por métodos parasitológicos.

Todos os pacientes apresentaram síndrome febril de início súbito, prolongada, sem periodicidade, inicialmente elevada, acompanhada frequentemente de fadiga, calafrios, cefaléia e mialgia. O tempo entre o início da doença até o início do tratamento foi de cerca de 30 dias. O período febril variou entre dois e 28 dias, durando, em média, 20 dias. As manifestações clínicas mais frequentes foram febre (100\%), fadiga (100\%), cefaléia ( $86,2 \%)$, mialgia $(82,8 \%)$, calafrios $(58,6 \%)$, palidez $(58,6 \%)$, dispnéia $(48,3 \%)$, edema de face $(34,5 \%)$, edema de membros inferiores/generalizado (31\%), dor abdominal $(31 \%)$, adenomegalia $(24,1 \%)$, hepatomegalia $(24,1 \%)$, exantema $(13,8 \%)$, icterícia $(10,3 \%)$, esplenomegalia $(6,9 \%)$, alterações do ritmo cardíaco (com bloqueio de ramo direito no ECG) (6,9\%) e meningoencefalite $(3,4 \%)$. Nenhum óbito relacionado à doença de Chagas aguda ocorreu para esta série de casos.

Nenhum paciente apresentou chagoma de inoculação. Quatro apresentaram sinal de Romaña, sendo que nenhum fazia parte de surtos. Para a maioria dos casos não foi possível determinar a forma de transmissão, mas não existe histórico de transfusão, transplante de órgãos ou acidentes de laboratório para nenhum dos casos, o que exclui estas formas de aquisição da doença.

No presente trabalho, verificou-se que a doença de Chagas aguda incidiu com frequência em indivíduos jovens, com 51,7\% dos casos em menores de 21 anos, predominando no sexo masculino. Em Bambuí, Estado de Minas Gerais, área endêmica para doença de Chagas, entre 100 casos agudos estudados, apenas 5\% eram maiores de 21 anos $^{7}$. No Estado do Pará, a faixa etária predominante foi de adultos entre 18 e 59 anos, havendo predomínio do sexo feminino ${ }^{1}$.

Demonstrou-se a ocorrência da infecção chagásica aguda em municípios situados nas regiões norte e oeste do Estado do Amazonas. A concentração dos casos em Tefé, município às margens do Rio Solimões, deve-se à ocorrência de um surto, como citado anteriormente, cujas características sugerem que a transmissão foi oral. Um caso de Manaus possivelmente se infectou na região do Rio Purus, pois o mesmo apresentou sintomas pouco tempo depois de uma pescaria nesta área, destacando que por ocasião da investigação do caso foram encontrados dois exemplares machos de Rhodnius pictipes no porão do seu barco. Para a maioria dos municípios onde ocorreram os casos, não existem informações sobre a fauna triatomínica. Investigações realizadas em Manaus evidenciaram o predomínio das espécies $R$. pictipes, $R$. robustus e Panstrongylus geniculatus ${ }^{8}$, que são frequentemente encontradas com altas taxas de infecção natural pelo T. cruzi em diversos ecótopos naturais na Amazônia Brasileira, onde esporadicamente invadem as residências ${ }^{9}$. No município de São Paulo de Olivença, na região do Alto Solimões, onde foi registrado o primeiro caso de doença de Chagas do Estado do Amazonas, R. pictipes foi a espécie predominante no intradomicílio ${ }^{10}$.

As características das habitações observadas para os casos facilitam o contato homem-vetor, assemelhando-se aos encontrados em outras áreas da Amazônia Brasileira ${ }^{1}$. Na casuística de Miles cols ${ }^{11}$, no Estado do Pará, de suposta transmissão silvestre, as condições de moradia, especialmente casas da zona rural construídas em madeira e telha em áreas de várzea, demonstram a provável exposição dos indivíduos estudados ao contato com vetores peridomiciliares.

Uma constante no peridomicílio dos casos rurais é a presença de palmeiras das espécies Maximiliana maripa (inajá) na região do Rio Negro, Attalea phalerata (urucuri) nas Regiões do Solimões, Purus e Juruá, Orbignya phalerata (babaçu) no Rio Negro e Juruá, Oenocarpus bataua (patauá) e Oenocarpus bacaba (bacaba), no Rio Negro e Solimões. Nestas palmeiras, podem se refugiar roedores, marsupiais e outras fontes alimentares para os triatomíneos que formam seus criadouros neste ecótopo.

Verificou-se um elevado percentual de positividade para as técnicas parasitológicas, especialmente a gota espessa. No contexto amazônico, este é um fator importante, já que a vigilância da doença de Chagas aguda nesta região está atrelada à da malária. No Estado do Pará, os testes empregados para o diagnóstico também mostraram boa sensibilidade para detecção dos casos agudos, apesar de menor que a verificada neste trabalho, com percentuais de positividade de $46,5 \%, 47,5 \%, 57,1 \%, 61,6 \%$ e $86,7 \%$ para exames de hemocultura, gota espessa ou exame direto, $\mathrm{QBC}$, xenodiagnóstico e pesquisa de IgM antiT. cruzi, respectivamente. Na Venezuela, foram encontrados percentuais de positividade de $34 \%, 61 \%$ e $53 \%$, para gota espessa, xenodiagnóstico e hemocultura, respectivamente ${ }^{12}$. Pinto cols ${ }^{1}$ chamaram a atenção de que indivíduos com parasitemia evidente em sangue periférico apresentaram resultados de xenodiagnósticos ou hemoculturas negativos em percentuais consideráveis, fato que não observamos neste grupo de pacientes.

A febre foi detectada na totalidade dos casos apresentados neste trabalho, concordando com pesquisa realizada em Bambuí, Estado de Minas Gerais ${ }^{13}$ e no Estado do Pará, onde a freqüência dos outros sinais e sintomas também é semelhante à desta casuística ${ }^{1}$. De um total de 233 pacientes estudados no Pará por Pinto cols ${ }^{1}, 13$ (5,6\%) foram a óbito na vigência da fase aguda, principalmente devido à miocardite aguda (46,2\%). Em Teutônia, no Rio Grande do Sul, o quadro clínico foi consideravelmente diferente, principalmente quanto à gravidade dos casos, especialmente em relação ao comprometimento cardíaco e a letalidade, ocorrida em $35,3 \%$ dos pacientes ${ }^{14}$. Nenhum óbito foi registrado nesta casuística. Entre os casos relatados nesta série, destaca-se o de uma paciente de 16 anos, procedente de Tefé, integrante do surto de 2004, que iniciou com síndrome febril aguda e evoluiu com meningoencefalite ${ }^{15}$.

Uma das prováveis vias de transmissão da doença de Chagas na Amazônia pode explicar, em parte, a apresentação da doença quase sempre aparente e polissintomática, mas com relativa benignidade de prognósticos imediatos ${ }^{1}$. Os indivíduos que se contaminam por via oral, dependendo da quantidade do inóculo, podem evoluir com infecções parasitárias maciças ou eliminar o parasito, antes mesmo que ele possa causar a infecção. Entretanto, inóculos maiores ou cepas mais agressivas podem determinar doença mais grave.

Em face ao crescente interesse que a doença de Chagas vem despertando na Amazônia, as informações sobre aspectos epidemiológicos da dinâmica da doença que proporcionem o entendimento e a ampliação dos conhecimentos sobre o registro da ocorrência de casos ou surtos na região são de grande relevância. Destaca-se a necessidade de estudos sistematizados de seguimento dos casos agudos que possam avaliar evoluções e respostas ao tratamento. 


\section{AGRADECIMENTOS}

À Gerência de Entomologia da Fundação de Medicina Tropical do Amazonas pela manutenção dos arquivos com a informação dos pacientes com doença de Chagas atendidos nesta instituição.

\section{CONFLITO DE INTERESSE}

Os autores declaram não haver nenhum tipo de conflito de interesse no desenvolvimento do estudo.

\section{SUPORTE FINANCEIRO}

Fundação de Medicina Tropical do Amazonas.

\section{REFERÊNCIAS}

1. Pinto AYN, Valente SA, Valente VC, Ferreira-Junior AG, Coura JR. Fase aguda da doença de Chagas na Amazônia brasileira. Estudo de 233 casos do Pará, Amapá e Maranhão observados entre 1988 e 2005. Rev Soc Bras Med Trop 2008; 41:602614.

2. Ministério da Saúde. Casos de Doença de Chagas Aguda (DCA). Brasil, Grandes Regiões e Unidades Federadas, 2005 a 2009. Disponível em http:// portal.saude.gov.br/portal/arquivos/pdf/tabchagascasos0509.pdf (acessado em 05/10/09).

3. Camargo ME, Silva GR, Castilho EA, Silveira AC. Inquérito sorológico da prevalência da infecção chagásica no Brasil, 1975-1980. Rev Inst Med Trop São Paulo 1984; 26;192-204.

4. Albajar PV, Laredo SV, Terrazas MB, Coura JR. Miocardiopatia dilatada em pacientes com infecção chagásica crônica. Relato de dois casos fatais autóctones do Rio Negro, Estado do Amazonas. Rev Soc Bras Med Trop 2003; 36;401407.

5. Xavier SS, Souza AS, Viñas PA, Junqueira ACV, Bóia MN, Coura JR. Cardiopatia chagásica crônica no Rio Negro, Estado do Amazonas. Relato de três novos casos autóctones, comprovados por exames sorológicos, clínicos, radiográficos do tórax, eletro e ecocardiográficos. Rev Soc Bras Med Trop 2006; 39:211-216.

6. Magalhães BML. Doença de Chagas no Amazonas: Prevalência sorológica em área rural de Manaus. Dissertação de Mestrado, Universidade do Estado do Amazonas, Manaus, AM; 2009.

7. Laranja FS, Dias E, Nobrega G. Clinica y terapeutica de la enfermedad de Chagas. Prensa Med Argent 1951; 38:465-484.

8. Magalhães LKC, Prestes SR, Fé NF, Ciriano CM, Fé FAA, Fabiano MP, Maciel MG, Oliveira JC, Silveira H, Barbosa MGV. Infecção natural e diversidade de espécies de vetores de doença de Chagas nos municípios de Manaus, Coari e Tefé/AM. Rev Soc Bras Med Trop 2009; 42:156.

9. Aguilar HM, Abad-Franch F, Dias JCP, Junqueira ACV, Coura JR. Chagas disease in the Amazon Region. Mem Inst Oswaldo Cruz 2007; 102:47-55.

10. Fé NF, França MS, Carvalho-Costa FA. Reassessing the entomological investigation around the first autochthonous case of Chagas disease in Western Brazilian Amazon. Mem Inst Oswaldo Cruz 2009; 104:121-123.

11. Miles MA, Souza AA, Póvoa MM. Chagas'disease in the Amazon Basin. III. Ecotopes of ten triatomine bug species (Hemiptera: Reduviidae) from the vicinity of Belém, Pará State. Braz J Med Entomol 1981; 18:266-278.

12. Parada H, Carrasco HA, Añez N, Fuenmayor C, Inglessis I. Cardiac involvement is a constant finding in acute Chagas'disease: a clinical, parasitological and histopathological study. Int J Cardiol 1997; 60:49-54.

13. Dias JCP. Doença de Chagas em Bambuí, Minas Gerais, Brasil. Estudoepidemiológico a partir da fase aguda, ente 1940 a 1982 . Tese de Doutorado, Universidade Federal de Minas Gerais, Belo Horizonte; 1982.
14. Silva NN, Clausel DT, Nolibus H, Mello AL Ossanai J, Rapone T, Snell T. Surto epidêmico de doença de Chagas com provável contaminação oral. Rev Inst Med Trop São Paulo 1968;10:265-276.

15. Medeiros MB, Guerra JAO, Lacerda MVG. Meningoencephalitis in a patient with acute Chagas disease in the Brazilian Amazon. Rev Soc Bras Med Trop 2008; 41:520-521. 